\title{
Linewidth broadening and emission saturation of a resonantly excited quantum dot monitored via an off-resonant cavity mode
}

Ulhaq, A.; Ates, Serkan; Weiler, S.; Ulrich, S.M.; Reitzenstein, S.; Löffler, A.; Höfling, S.; Worschech, L.; Forchel, A.; Michler, P.

Published in:

Physical Review B Condensed Matter

Link to article, DOI:

10.1103/PhysRevB.82.045307

Publication date:

2010

Document Version

Publisher's PDF, also known as Version of record

Link back to DTU Orbit

Citation (APA):

Ulhaq, A., Ates, S., Weiler, S., Ulrich, S. M., Reitzenstein, S., Löffler, A., Höfling, S., Worschech, L., Forchel, A., \& Michler, P. (2010). Linewidth broadening and emission saturation of a resonantly excited quantum dot monitored via an off-resonant cavity mode. Physical Review B Condensed Matter, 82(4), 045307. https://doi.org/10.1103/PhysRevB.82.045307

\section{General rights}

Copyright and moral rights for the publications made accessible in the public portal are retained by the authors and/or other copyright owners and it is a condition of accessing publications that users recognise and abide by the legal requirements associated with these rights.

- Users may download and print one copy of any publication from the public portal for the purpose of private study or research.

- You may not further distribute the material or use it for any profit-making activity or commercial gain

- You may freely distribute the URL identifying the publication in the public portal 


\title{
Linewidth broadening and emission saturation of a resonantly excited quantum dot monitored via an off-resonant cavity mode
}

\author{
A. Ulhaq, ${ }^{1, *}$ S. Ates, ${ }^{2}$ S. Weiler, ${ }^{1}$ S. M. Ulrich, ${ }^{1}$ S. Reitzenstein, ${ }^{3}$ A. Löffler, ${ }^{3}$ S. Höfling, ${ }^{3}$ L. Worschech,,${ }^{3}$ \\ A. Forchel, ${ }^{3}$ and P. Michler ${ }^{1}$ \\ ${ }^{1}$ Institut für Halbleiteroptik und Funktionelle Grenzflächen, Universität Stuttgart, Allmandring 3, 70569 Stuttgart, Germany \\ ${ }^{2}$ Department of Photonics Engineering, Technical University of Denmark, 2800 Kgs Lyngby, Denmark \\ ${ }^{3}$ Technische Physik, Universität Würzburg, Am Hubland, 97074 Würzburg, Germany
}

(Received 14 May 2010; published 12 July 2010)

\begin{abstract}
We report on the robustness of a detuned mode channel for reading out the relevant $s$-shell properties of a resonantly excited coupled quantum dot (QD) in a pillar microcavity. The line broadening of the QD $s$-shell is "monitored" by the mode signal with high conformity to the directly measured QD linewidth. The mode signal also monitors the saturation behavior of a near Fourier transform-limited photon emission from a resonantly excited QD. We also investigate the temperature dependence of the coupling mechanism between an offresonant QD and a cavity mode under pure resonant excitation of the quantum emitter.
\end{abstract}

DOI: 10.1103/PhysRevB.82.045307

PACS number(s): 78.67.Hc, 42.50.Dv, 42.50.St, 78.55.-m

Non-resonant coupling is an interesting phenomenon recently observed by many groups for QDs in high quality factor $(Q \geq 10000)$ optical microcavities. ${ }^{1-3}$ The effect realizes the fact that a solid state quantum emitter, like a $\mathrm{QD}$, is fundamentally different from a simple two-level system used in standard atom optics models. ${ }^{4}$ Enhanced cavity mode emission is observed even for QDs detuned by a few meV from a coupled cavity mode. ${ }^{5}$ Several recent studies explained this effect as a result of pure dephasing in such solid state systems. ${ }^{6,7}$ More specifically, the important role of pure dephasing caused by coupling to the phonon bath of the solid state environment of the QD was highlighted. ${ }^{8-11}$ The effect present at detunings larger than $1 \mathrm{meV}$ was also proposed to be a result of photon-induced "shake up" processes mainly due to the coupling of quasicontinuum excited states of the QD to the cavity modes. ${ }^{12}$ This nonresonant coupling effect gains even more importance under pure resonant excitation of a quantum dot. The cavity mode channel is not only enhanced under $s$-shell excitation of the QD due to the nonresonant coupling effect, but is also capable to "read out" relevant $s$-shell properties such as emission line width, exciton fine structure splitting, emission saturation and photon statistics of the coupled QD as shown by Ates et al. ${ }^{9}$ This read-out scheme via nonresonant QD-cavity coupling has the advantage that the mode signal is background-free as it is spectrally detuned from the $s$-shell and hence no scattered laser photons are collected. This softens the strict experimental criterion of separating the laser signal from QD fluorescence for investigations of relevant $s$-shell properties.

In this work we demonstrate that the enhanced cavity channel under $s$-shell excitation can be further used to "monitor" the effect of power broadening of the exciton resonance with high conformity between the QD and mode profiles. Using the cavity mode channel, we also monitor the saturation behavior of nearly Fourier transform-limited photon emission from the coupled quantum dot. We investigate the possible role of phonon-assisted pure dephasing by systematic temperature-dependent $s$-shell resonance scans, thereby minimizing any possible influence of the quasicontinuum of excited states of the quantum dots in the coupling mechanism. ${ }^{12}$
Self-assembled In(Ga)As/GaAs quantum dots grown by molecular beam epitaxy are used in this study. The QDs are embedded as a single active layer at the center between top and bottom distributed Bragg reflector (DBR) layers. The initial planar structure was etched into fields of micropillars ${ }^{13}$ with $1.75 \mu \mathrm{m}$ diameter, each containing about 100 QDs on average. For optical studies, the sample was held in a Helium-flow cryostat, where the temperature can be varied from $T=4 \mathrm{~K}$ to room temperature with a precision of $\pm 0.5 \mathrm{~K}$. A tunable Ti:sapphire $\mathrm{cw}$ ring laser, providing an emission full width at half maximum (FWHM) of $\sim 500 \mathrm{kHz}$, was used to selectively and resonantly excite individual QDs within single micropillars. The emission from micropillars was collected by a spectrometer-CCD combination with a spectral resolution of $\sim 35 \mu \mathrm{eV}$. Details of the experimental setup are given in Ref. 14 .

In the first part of our measurements, resonant fluorescence emission of single QDs within the micropillars has been investigated in dependence on temperature-controlled QD-mode spectral detuning. The pillar (Pillar 1) used in these measurement has fundamental mode (FM) emission at $E_{c}=1.3568 \mathrm{eV}$, with a linewidth (FWHM) of $\Delta E_{c}$ $=99.67 \mu \mathrm{eV}$, corresponding to a quality factor $Q$ $=13600 \pm 400$. The estimated cavity mode volume of the pillar is $V_{m} \approx 0.41 \mu \mathrm{m}^{3} \sim 25(\lambda / n)^{3}$. Based on these values, and under the assumption of perfect spatial overlap between emitter and cavity, we calculate a theoretical Purcell factor of 42. From time-resolved PL measurements (not shown) on a weakly coupled QD in the pillar (see discussion below), a pronounced Purcell enhancement factor of $F_{P} \approx 13$ was verified, being indicative of large QD-mode overlap.

The excitation laser was scanned over the $s$-shell of a single QD in steps of $\sim 250 \mathrm{MHz}$ at a constant excitation power of $P_{0}=300 \mathrm{nW}$. A full series of temperaturedependent PL spectra under successive resonance scanning over the QD $s$ shell is shown in Fig. 1(a)-1(f) as intensity plots. Figure 1(a) shows such a frequency scan taken at a sample temperature of $T=24 \mathrm{~K}$, where the QD exciton emission energy is at $1.3567 \mathrm{eV}$. The QD is red-detuned from the FM cavity mode by $\Delta E=E_{Q D}-E_{M o d e}=-150 \mu \mathrm{eV}$. The hori- 


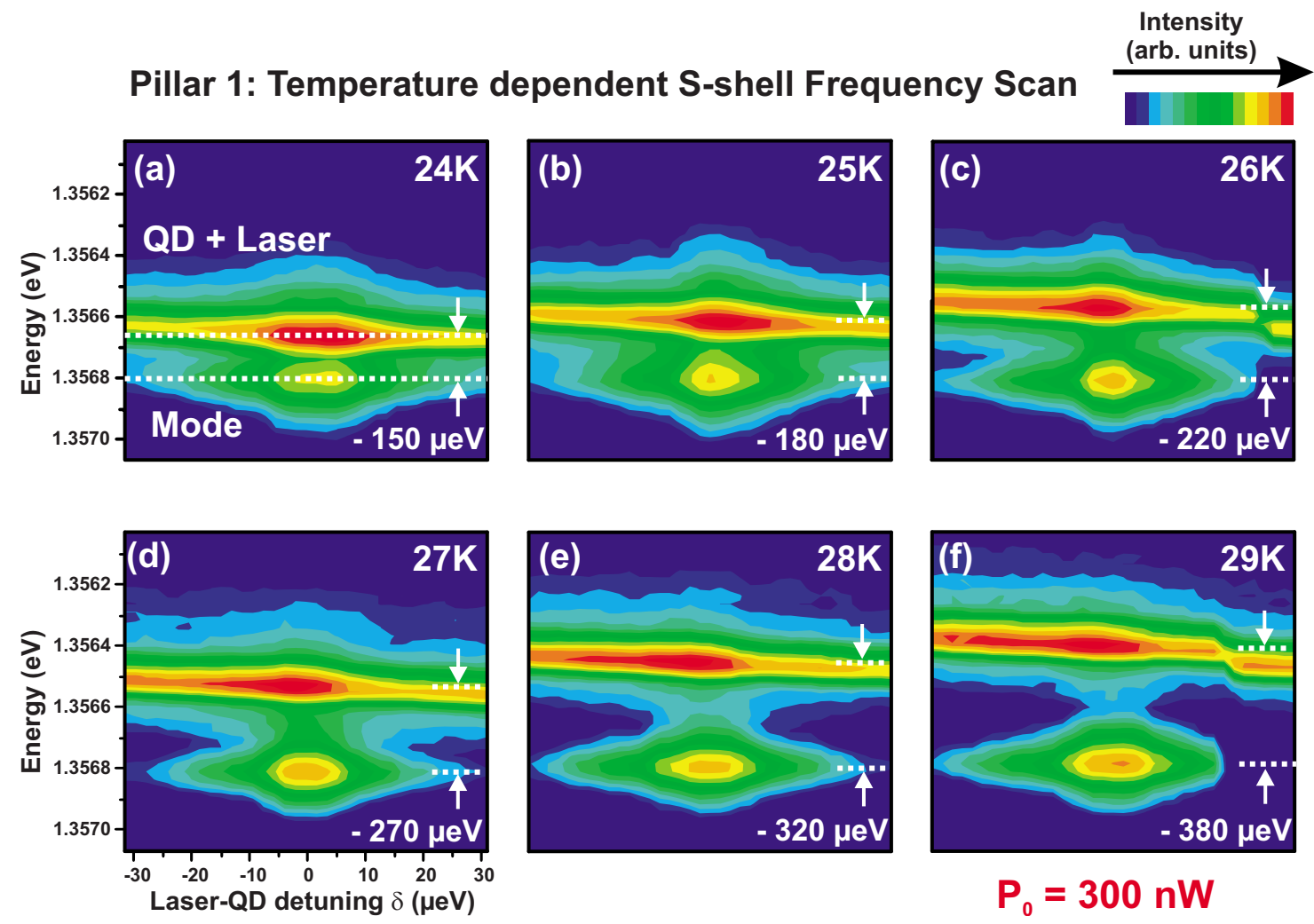

FIG. 1. (Color online) Temperature dependent resonance scans: (a)-(f) frequency scans of cw laser over the $s$-shell of a QD in Pillar 1, each obtained at different sample temperatures and hence QD-mode detunings $\Delta E(T)$. The FM emission of the pillar is around 1.3578 eV for all these scans. Each plot is a color scale of the intensity of spectra taken at gradually varying laser-QD detunings $\delta$. All of the frequency scans shown were performed at a fixed laser power of $P_{0}=300 \mathrm{nW}$. The composite QD and laser signal along with the cavity mode emission is clearly visible in all plots. Horizontal dashed lines mark the QD exciton and cavity mode positions at the given temperatures. The discontinuities in (c) and (f) are an artifact due to the laser mode-hop.

zontal dashed lines in the figure represent the QD exciton and cavity mode resonances, respectively. In all intensity plots of Fig. 1, the near resonant scattered laser signal, superimposed on the QD exciton emission, appears as a single line. The FM emission, distinctly detuned from the QD $s$-shell, appears as a separate signature. A significant increase in the composite QD and laser signal is observed under excitation within the QD $s$-shell resonance [where $\delta=\left(E_{\text {laser }}\right.$ $\left.-E_{Q D}\right) \rightarrow 0$ ], which clearly demonstrates the onset of resonance fluorescence from the excited QD. Parallel to the QD emission (resonance fluorescence), we simultaneously observe, an increase (decrease) of the spectrally separated mode signal as the laser is tuned in (out) of the narrow QD $s$-shell resonance. This cavity mode signal enhancement acts not only as a signature of laser-QD resonance but also as an indicator of coupling between a resonantly excited QD and the off-resonant cavity mode. ${ }^{9}$ This nonresonant coupling effect is consistently observed in all frequency scans, exhibiting the robustness and repeatability of the phenomenon for QD-mode detunings of up to $\sim 400 \mu \mathrm{eV}(30 \mathrm{~K})$.

Figure 2(a) shows the emission spectra observed at exact laser-QD resonance $\delta=0$ which have been extracted from the frequency scan series shown in Figs. 1(a) -1 (f) for various QD-cavity mode detunings $\Delta E$. Comparing the $\delta=0$ spectra at different temperature, the mode signal shows a clear enhancement with increased QD-mode detuning. For a quanti- (a) S-shell exc: Resonant Spectra

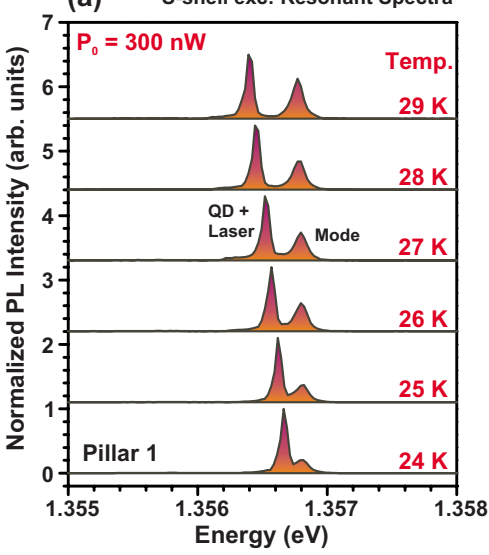

(b) Rel. Mode Intensity

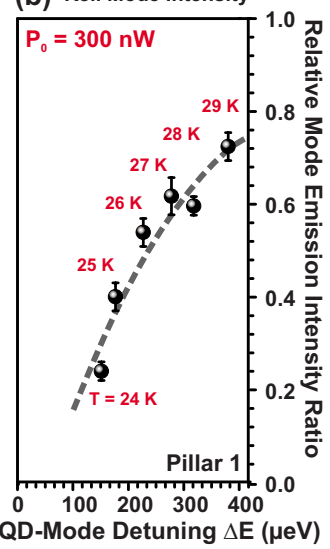

FIG. 2. (Color online) Temperature dependence of Relative Mode Intensity under $s$-shell excitation: (a) set of spectra at the exact laser-QD resonance position $(\delta=0)$ obtained from scans in Figs. 1(a)-1(f) at different temperatures $T$. The composite QD and laser line contains the resonance fluorescence signal from the QD while the blue detuned line corresponds to the enhanced cavity mode. The detuning between the resonantly excited QD and mode signal $\Delta E(T)$ gradually increases with increasing temperature. (b) Relative mode intensity, calculated from the spectra in (a), as a function of increasing QD-cavity mode detuning $\Delta E$. The corresponding temperatures are indicated in the figure. 
(a)

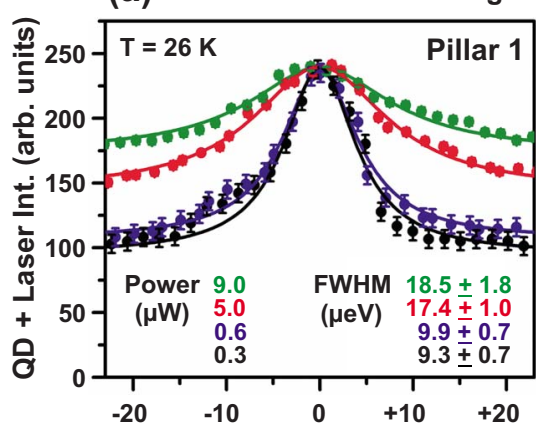

(b)

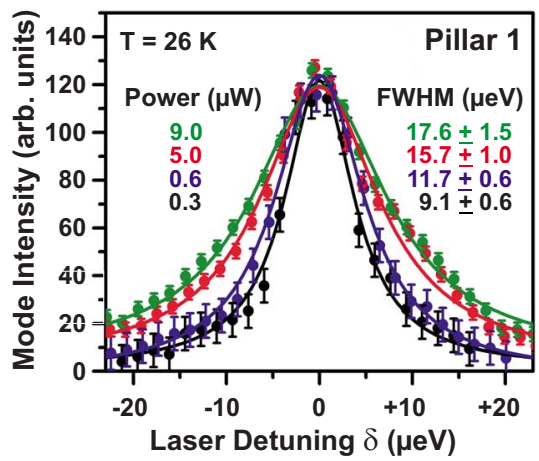

(c)

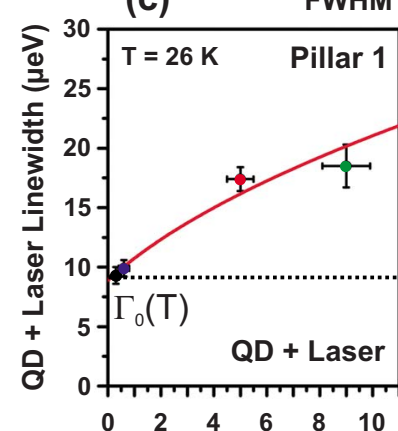

(d)

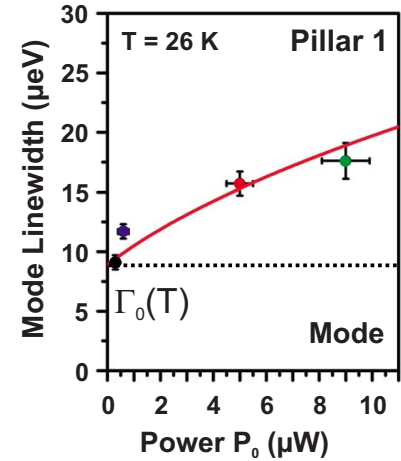

(e) Pillar 2: Frequency Scan

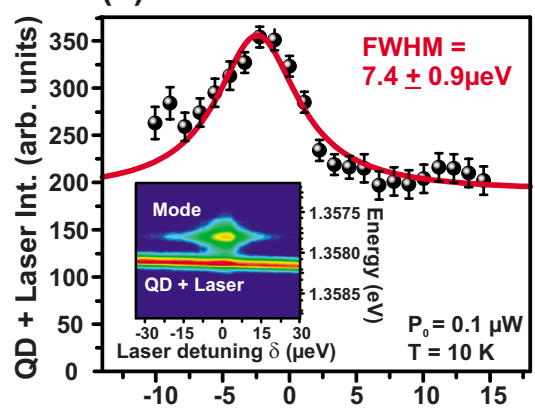

(f)

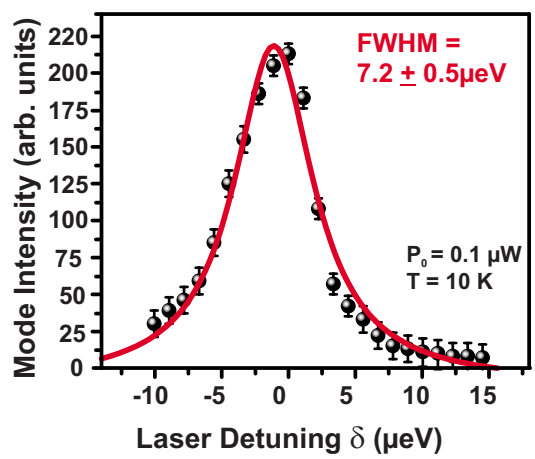

FIG. 3. (Color online) Power-dependent linewidth analysis of resonance scans: line shapes of the resonantly excited QD in Pillar 1, derived from the full frequency scans performed over the $s$-shell of the quantum dot. Scans were performed at increasing excitation powers at a constant sample temperature of $T=26 \mathrm{~K}$. (a) Composite QD and laser signal profiles derived from resonance scans at variable excitation powers. Solid lines are Lorentzian fits to the data. (b) The coupled cavity mode signal profile for the corresponding excitation powers. (c) FWHM extracted from line fits in (a) as a function of increasing excitation power. The solid line is a theoretical fit to the data. (d) FWHM calculated from (b) along with the fit (solid line) for the mode signal. (e) and (f) QD and mode emission profiles obtained from a frequency scan over the $s$-shell of another QD (Pillar 2). The corresponding frequency scan is shown in the inset of plot (e). The frequency scan in this case is performed at a constant laser excitation power of $P_{0}=100 \mathrm{nW}$.

tative analysis, we have calculated the relative integral mode intensity, defined as $I_{\text {rel }}^{M}=\int I_{\text {mode }} d \omega / \int\left(I_{\text {mode }}+I_{Q D}\right) d \omega$, from the spectra in Fig. 2(a). The quantum dot intensity is measured by subtracting the constant laser background from the composite QD and laser signal. The dependence of relative mode intensities $I_{r e l}^{M}$ on QD-cavity mode detuning $\Delta E$ is shown in Fig. 2(b). As is highlighted by the dashed guideline to the data, a clear increase in $I_{r e l}^{M}$ is observed as the QD is further detuned from the cavity mode under increasing temperatures. The decrease in $I_{r e l}^{M}$ near QD-mode resonance under pure QD $s$-shell excitation is consistent with results reported recently under quasiresonant $p$-shell excitation ${ }^{9}$ and possibly points toward a major role of acoustic phononbased pure dephasing ${ }^{8}$ as the effective coupling mechanism between the QD and the remote FM mode channel.

To further investigate the robustness of the mode channel as a suitable monitor of QD $s$-shell emission characteristics under purely resonant excitation, $s$-shell frequency scans have additionally been performed as a function of laser power on Pillar 1. The excitation power was gradually increased from $P_{0}=0.3-10 \mu \mathrm{W}$ at a constant QD-mode detuning of $\Delta E=-220 \mu \mathrm{eV}$. The profiles of the resulting composite QD and laser line, as well as the cavity mode line, are presented in Figs. 3(a) and 3(b), respectively. Each data point in the plot represents the integrated intensity of the QD and laser or mode line at the corresponding laser-QD detuning $\delta$ and excitation power. The data points are fitted with Lorentzian profiles, with the extracted QD exciton linewidths (FWHM) indicated in the figure. An increase in the FWHM linewidth is observed in parallel for both signal profiles with increasing excitation power. This well known phenomenon of power broadening is indeed expected under strictly resonant $\delta=0$ excitation of a two-level system. The absorption saturates within the narrow homogeneous linewidth of the absorption profile even at lower excitation powers under resonant excitation. Thus, high excitation powers considerably increase the absorption in the branches of the absorption profile, which effectively broadens the spectral resonance profile. $^{15}$

Figures 3(c) and 3(d) depict the observed resonance profile linewidths as an explicit function of excitation power together with a theoretical fit according to the linewidth expected for a resonantly driven two-level system. ${ }^{17,18}$ The resonant FWHM $\Gamma\left(P_{0}\right)$ as a function of laser power $P_{0}$ can be expressed as

$$
\Gamma\left(P_{0}, T\right)=\Gamma_{0}(T) \sqrt{1+\alpha P_{0}}
$$

with $\alpha$ as a proportionality factor between excitation power and Rabi frequency $\left(\Omega^{2}=\alpha P_{0}\right) \cdot{ }^{17}$ Consistent values of 

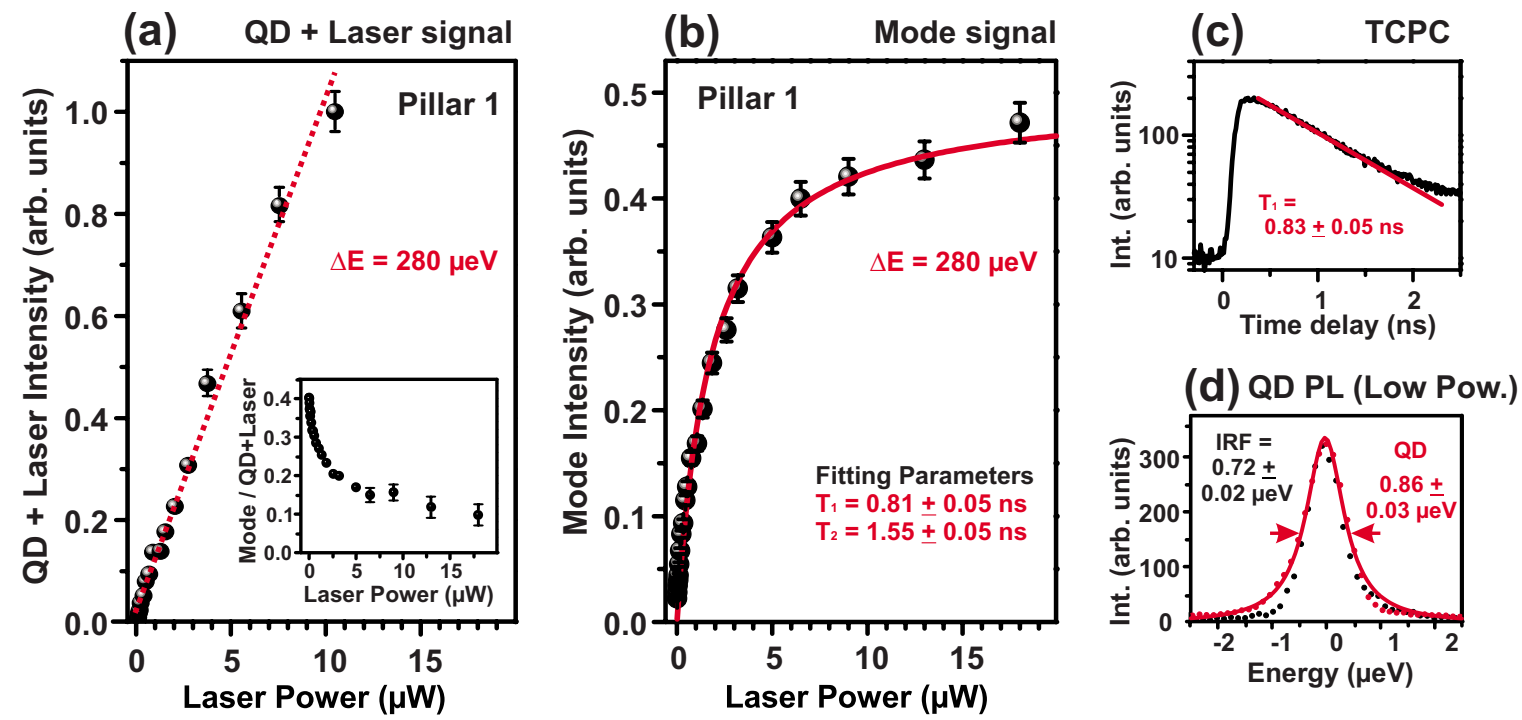

FIG. 4. (Color online) Saturation of nearly Fourier transform-limit photon emission from resonantly excited QD: (a) integrated intensity of the composite QD and laser emission as a function of increasing excitation power $P_{0}$ under $s$-shell excitation of the QD at $T=9.5 \mathrm{~K}$. The inset shows the ratio of the detuned mode signal to the composite QD and laser signal at the corresponding laser powers. The red dashed line is a guide to the eyes. (b) The integrated intensity of the coupled cavity mode as a function of increasing excitation power. The solid red line is a theoretical fit to the data points using the fitting parameters of $T_{1}=0.81 \pm 0.05 \mathrm{~ns}$ and $T_{2}=1.55 \pm 0.05$ ns. (c) Time correlated photon counting (TCPC) measurement of the QD exciton under $p$-shell excitation at $T=9.5 \mathrm{~K}$. (d) High resolution PL spectrum of the QD emission (red points) under $s$-shell excitation performed at $P_{0}=1.0 \mu \mathrm{W}$. The red line is a Lorentzian fit to the data while the black points in represents the PL of the $\mathrm{cw}$ laser representing the instrumental response function (IRF) of the setup i.e., $0.72 \pm 0.02 \mu \mathrm{eV}$.

$\Gamma_{0}(T)=2 \hbar / T_{2}=8.9 \pm 0.2 \mu \mathrm{eV}$ are derived as the low power FWHM limit of $s$-shell resonance in both the QD and mode emission signals at $T=26 \mathrm{~K}$. Due to the elevated temperature applied in these measurements, even the low power resonance profile $\Gamma_{0}(T)$ of the QD is expected to be initially broadened by phonon dephasing. ${ }^{19}$

It is important to note that the linewidths derived from the QD resonance enhancement are in high conformity with those obtained from the mode enhancement profile. We like to emphasize that this conformance was consistently observed in our micropillar samples. For comparison, we show a QD resonance signal and an enhanced cavity mode profile of another micropillar (Pillar 2) in Figs. 3(e) and 3(f). Single QD exciton for this pillar appears at $1.3581 \mathrm{eV}$ while the FM emission of the pillar is at $1.3578 \mathrm{eV}$ at a sample temperature of $10 \mathrm{~K}$. Under these conditions, the QD is blue detuned from the cavity mode by $\Delta E=+328 \mu \mathrm{eV}$. The corresponding resonance scan is shown as an inset in Fig. 3(e). The linewidths derived from the QD and cavity mode profiles are again in high correspondence with each other. We would like to emphasize that this is in contrast with a recent study, ${ }^{16}$ where a coupled cavity mode channel revealed broadened linewidths as compared to the QD resonance profile for photonic crystal nanocavities. The effect was attributed to the supposed presence of additional dephasing processes involved in the energy transfer between the excited QD and the detuned cavity mode. Our results on the contrary indicate minimal presence of such extra dephasing mechanisms in our micropillar systems. This observation may be an indication of inherent differences with respect to the coherent energy transfer mechanisms between a QD and a detuned cavity in these types of cavity systems.
Additional measurements have addressed the explicit power saturation behavior of single QDs under strictly resonant $s$-shell excitation $(\delta=0)$. Spectra of Pillar 1 were taken at gradually increased laser powers for a constant QD-mode detuning of $\Delta E=-280 \mu \mathrm{eV}(T=9.5 \mathrm{~K})$. Integrated intensities of the QD and laser signal and the detuned mode signal are calculated from the spectra and plotted separately in Figs. 4(a) and 4(b), respectively. The composite signal in [plot (a)] shows an almost linear increase while the background-free mode signal [plot (b)], on the other hand, reveals a clear saturation behavior with increasing excitation power. Powerdependent saturation of a resonantly excited two-level system is a well known phenomenon. ${ }^{15}$ The absence of saturation in the composite QD and laser signal can be attributed to the increasing scattered laser signal which overcomes and finally dominates the resonance fluorescence signal at higher excitation powers. This fact is nicely demonstrated in the inset of Fig. 4(a), where a near exponential decrease in the ratio between cavity mode and composite QD and laser signal reflects the increasing contribution of the scattered laser signal. On the other hand, the saturation of the emitter is nicely monitored by the coupled FM cavity signal due to the absence of laser background. The data points are fitted using a theoretical model for a resonantly pumped two-level system under zero laser detuning $(\delta=0) .{ }^{18,20}$ The spontaneous emission rate $\left(I_{\text {res }}\right)$ of such a system obeys the relation

$$
I_{r e s}\left(P_{0}\right) \propto \frac{1}{2} \frac{\Omega^{2} \cdot T_{1} / T_{2}}{T_{2}^{2}+\Omega^{2} \cdot T_{1} / T_{2}},
$$

where the excitation power substitutes the effective Rabi frequency given by the relation $\Omega^{2}=\alpha P_{0}$. The above expression 
suggests that emission saturation is expected for a regime of strong excitation where $\Omega \gg 1 / \sqrt{T_{1} T_{2}}$. We obtain best fit values of $T_{1}=809 \pm 50 \mathrm{ps}$ for the radiative lifetime and $T_{2}$ $=1547 \pm 50 \mathrm{ps}$ for the emission coherence. The value of life time $T_{1}$ used as a fitting parameter is in high conformity with the experimentally verified value of $830 \pm 50$ ps from timeresolved PL measurement (TCPC) shown in Fig. 4(c). The emission coherence time was also independently measured from a high-resolution PL (HRPL) spectrum of the QD under $s$-shell excitation at low excitation power of $\sim 1.0 \mu \mathrm{W}$ [Fig. 4(d)]. The measured FWHM of the QD emission (red points and Lorentzian line fit) is $0.86 \pm 0.03 \mu \mathrm{eV}$, corresponding to a coherence time of $T_{2}=1530 \pm 35 \mathrm{ps}$. Again high correspondence with the fitting parameter used in Fig. 4(b). The HRPL spectrum of the narrow-band cw laser emission (black points) in the plot denotes the instrument response function (IRF) of the high-resolution setup, i.e., $0.72 \mu \mathrm{eV}$. Therefore, we conclude that the $T_{2} /\left(2 T_{1}\right)$ ratio we obtain for the pillar is $\sim 0.92 \pm 0.02$, indicating photon emission close to the Fourier transform-limit $\left[T_{2} /\left(2 T_{1}\right) \rightarrow 1\right]$ from this particular quantum dot. ${ }^{14}$

In conclusion, the presented work highlights the importance of nonresonant quantum dot-cavity coupling as a versatile and powerful tool to monitor important aspects of resonance fluorescence of a single quantum dot. The phe- nomenon allows to investigate in detail the excitation powerinduced linewidth broadening and emission saturation of a single QD photon source. These properties of a resonantly excited QD might otherwise be difficult to access due to the scattered resonant laser signal. Furthermore, we show that the monitoring process is not affected by the presence of additional dephasing mechanisms and the information extracted from the detuned mode channel are in high conformity with the resonantly excited QD signal. The work also reinforces the possible major contribution of phononmediated pure dephasing to the coupling mechanism between a QD exciton and an off-resonant high- $Q$ cavity mode. This work opens up the possibility of using a coupled cavity mode as a possible single-photon source as recently suggested, ${ }^{7}$ which can be of importance in the context of stable nonclassical quantum emitters for quantum information processing.

We thank M. Emmerling and A. Wolf for expert sample preparation. We gratefully acknowledge financial support from Deutsche Forschungsgemeinschaft (DFG) via the research group "Quantum optics in semiconductor nanostructures." A. Ulhaq acknowledges funding from International Max Planck Research School IMPRS-AM. S. Weiler acknowledges financial support by the Carl Zeiss Stiftung.
*Corresponding author; http://www.ihfg.uni-stuttgart.de; ata.ulhaq@ihfg.uni-stuttgart.de

${ }^{1}$ K. Hennessy, A. Badolato, M. Winger, D. Gerace, M. Atatüre, S. Gulde, S. Fält, E. L. Hu, and A. Imamoğlu, Nature (London) 445, 896 (2007).

${ }^{2}$ D. Press, S. Götzinger, S. Reitzenstein, C. Hofmann, A. Löffler, M. Kamp, A. Forchel, and Y. Yamamoto, Phys. Rev. Lett. 98, 117402 (2007).

${ }^{3}$ A. Badolato, M. Winger, E. L. Hu, and A. Imamoğlu, C. R. Phys. 9, 850 (2008).

${ }^{4}$ J. M. Raimond and S. Haroche, Rev. Mod. Phys. 73, 565 (2001).

${ }^{5}$ M. Kaniber, A. Laucht, A. Neumann, J. M. Villas-Bôas, M. Bichler, M.-C. Amann, and J. J. Finley, Phys. Rev. B 77, 161303(R) (2008).

${ }^{6}$ A. Naesby, T. Suhr, P. T. Kristensen, and J. Mørk, Phys. Rev. A 78, 045802 (2008).

${ }^{7}$ A. Auffèves, J.-M. Gérard, and J.-P. Poizat, Phys. Rev. A 79, 053838 (2009).

${ }^{8}$ J. Suffczyński, A. Dousse, K. Gauthron, A. Lemaître, I. Sagnes, L. Lanco, J. Bloch, P. Voisin, and P. Senellart, Phys. Rev. Lett. 103, 027401 (2009).

${ }^{9}$ S. Ates, S. M. Ulrich, A. Ulhaq, S. Reitzenstein, A. Löffler, S. Höfling, A. Forchel, and P. Michler, Nat. Photonics 3, 724 (2009).

${ }^{10}$ U. Hohenester, A. Laucht, M. Kaniber, N. Hauke, A. Neumann,
A. Mohtashami, M. Seliger, M. Bichler, and J. J. Finley, Phys. Rev. B 80, 201311(R) (2009).

${ }^{11}$ G. Tarel and V. Savona, Phys. Rev. B 81, 075305 (2010).

${ }^{12}$ M. Winger, T. Volz, G. Tarel, S. Portolan, A. Badolato, K. J. Hennessy, E. L. Hu, A. Beveratos, J. Finley, V. Savona, and A. Imamoglu, Phys. Rev. Lett. 103, 207403 (2009).

${ }^{13}$ S. Reitzenstein, C. Hofmann, A. Gorbunov, M. Strauß, S. H. Kwon, C. Schneider, A. Löffler, S. Höfling, M. Kamp, and A. Forchel, Appl. Phys. Lett. 90, 251109 (2007).

${ }^{14}$ S. Ates, S. M. Ulrich, S. Reitzenstein, A. Löffler, A. Forchel, and P. Michler, Phys. Rev. Lett. 103, 167402 (2009).

${ }^{15}$ L. Allen and J. H. Eberly, Optical Resonance and Two-Level Atoms (Wiley, New York, 1975)

${ }^{16}$ A. Majumdar, A. Faraon, E. D. Kim, D. Englund, H. Kim, P. Petroff, and J. Vučković, preceding paper, Phys. Rev. B 82, 045306 (2010).

${ }^{17}$ S. Stufler, P. Ester, A. Zrenner, and M. Bichler, Appl. Phys. Lett. 85, 4202 (2004).

${ }^{18}$ A. Muller, E. B. Flagg, P. Bianucci, X. Y. Wang, D. G. Deppe, W. Ma, J. Zhang, G. J. Salamo, M. Xiao, and C. K. Shih, Phys. Rev. Lett. 99, 187402 (2007).

${ }^{19}$ M. Bayer and A. Forchel, Phys. Rev. B 65, 041308(R) (2002).

${ }^{20}$ E. B. Flagg, A. Muller, J. W. Robertson, S. Founta, D. G. Deppe, M. Xiao, M. Wa, G. J. Salamo, and C. K. Shih, Nat. Phys. 5, 203 (2009). 